\title{
Noninvasive and Invasive Assessments of the Functional Significance of Intermediate Coronary Artery Stenosis: Is This a Matter of Right or Wrong?
}

\author{
Fan Yongzhen ${ }^{\mathrm{a}, \mathrm{c}}$ Ki-Hyun Jeon ${ }^{\mathrm{a}}$ Joon-Hyung Doh ${ }^{\mathrm{b}}$ Bon-Kwon Koo ${ }^{\mathrm{a}}$ \\ ${ }^{a}$ Department of Medicine, Seoul National University Hospital, Seoul, and bepartment of \\ Medicine, Inje University Ilsan Paik Hospital, Goyang, Korea; ' Xiangtan Central Hospital, \\ Changde, China
}

\section{Key Words}

Coronary artery disease · Coronary computed tomographic angiography · Invasive coronary angiography · Intravascular ultrasound · Functional fractional reserve

\begin{abstract}
Coronary computed tomographic angiography (CCTA) has emerged in recent years as a noninvasive modality to evaluate coronary artery disease. Many studies have revealed the excellent diagnostic accuracy of CCTA when compared with invasive coronary angiography (ICA) or intravascular ultrasound (IVUS). It is generally accepted that the functional significance of coronary artery stenosis is important to make treatment decisions; however, CCTA, ICA, and IVUS are limited in the ability to determine the physiologic significance of coronary stenosis. The FIGURE-OUT study compared the noninvasive and invasive assessment of the functional significance of intermediate coronary artery stenosis.

(C) 2014 S. Karger AG, Basel
\end{abstract}

Recent advances in coronary computed tomographic angiography (cCTA) technology have improved its diagnostic performance of identifying anatomically significant coronary artery stenosis when compared with invasive coronary angiography (ICA) or intravascular ultrasound (IVUS) [1, 2]. A meta-analysis of 28 studies involving 1,286 patients that investigated the diagnostic performance of cCTA for the detection of coronary stenosis $>50 \%$, using

F.Y. and K.-H.J. contributed equally to this work. 

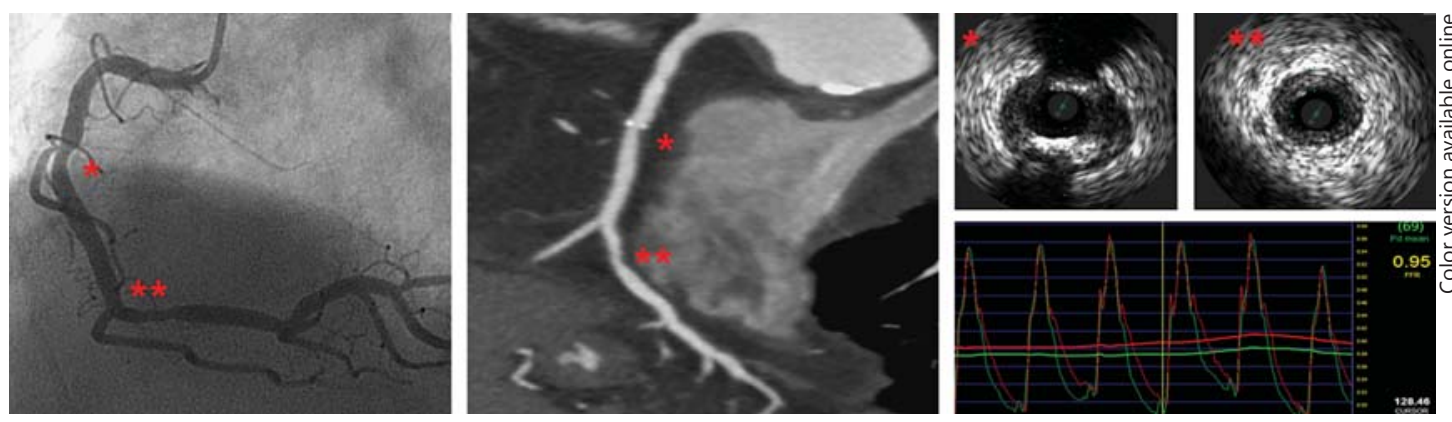

Fig. 1. Case example. By invasive angiography (left), two intermediate stenoses at the mid $\left({ }^{*}\right)$ and distal $\left({ }^{* *}\right)$ right coronary artery were found. cCTA (middle) shows the plaque as well as the luminal narrowing of those stenoses, and IVUS (upper right) provides precise quantitative and qualitative information. FFR (lower right) reveals that both stenoses are functionally insignificant and revascularization is not necessary.

ICA as the gold standard, showed a per-patient sensitivity of $99 \%$, a specificity of $89 \%$, a positive predictive value of $93 \%$, and a negative predictive value of $100 \%$ [3]. As cCTA has become one of the most commonly used noninvasive diagnostic tests for coronary artery disease (CAD), it is important to understand the relationship between CCTA, ICA, and IVUS parameters, as well as the diagnostic accuracy of cCTA compared with that of ICA and IVUS to define the functional significance of coronary stenosis (fig. 1).

Fractional flow reserve (FFR) represents the proportion of reduced myocardial blood flow due to stenosis to the normal maximal myocardial flow. Previous studies have shown the clinical benefits of FFR-guided revascularization. In the DEFER study, FFR was used to determine the need for percutaneous coronary intervention in intermediate stenosis, and it was found that for functionally insignificant lesions (FFR $\geq 0.75$ ), percutaneous coronary intervention did not improve the prognosis of the patients $[4,5]$. Later, the FAME I and FAME II randomized multicenter studies demonstrated the benefit of the FFR-guided revascularization strategy $[6,7]$. As a result, FFR is now regarded as the gold standard invasive method for determining the functional significance of intermediate stenosis in the cardiac catheterization laboratory, and it was classified as a Class IA recommendation in the 2010 European Guidelines on myocardial revascularization [8].

Although it is well known that cCTA can provide excellent anatomical information on obstructive CAD, previous studies have shown the variability in agreement between cCTA and ICA/IVUS parameters. Not only was there a discrepancy in previous results, but the degree of over- or underestimation of lesion severity was different among these studies [9, 10]. Furthermore, previous reports have suggested that the anatomical assessment by cCTA might have limitations in the evaluation of the functional significance of the stenoses, especially in intermediate stenoses $[11,12]$. This can precipitate unnecessary ICA and coronary intervention in patients who do not have functionally significant coronary stenosis causing myocardial ischemia.

The prospective multicenter FIGURE-OUT study was performed to determine the diagnostic value of cCTA in the assessment of the functional significance of intermediate stenosis as compared with that of ICA and IVUS [13]. There was a statistically significant but weak correlation between cCTA percent diameter stenosis (\%DS) and FFR ( $r=-0.271, p<0.001)$ and also between cCTA minimal lumen area (MLA) and FFR $(r=0.547, p<0.001)$. The correlations between ICA \%DS and FFR and between IVUS MLA and FFR ( $\mathrm{r}=-0.536, \mathrm{p}<$ $0.001 ; r=0.547, p<0.001$, respectively) were better, but only of moderate degree (fig. 2 ). These trends were consistent in different lesion subsets. The diagnostic performance of 
Yongzhen et al.: Noninvasive and Invasive Assessments of the Functional Significance of Intermediate Coronary Artery Stenosis: Is This a Matter of Right or Wrong?



Fig. 2. Relationship between FFR and MLA by cCTA and IVUS. The correlation between IVUS MLA and FFR (right) was better than that between cCTA MLA and FFR (left).

cCTA to define the functional significance of coronary stenosis by the receiver operating characteristic curve analysis was consequently lower than that of ICA and IVUS (area under the curve, cCTA \%DS vs. ICA \%DS, 0.657 vs. 0.765 ; cCTA MLA vs. IVUS MLA, 0.712 vs. 0.801 ). However, the diagnostic accuracy of these values to predict the presence of ischemia was between 60 and 70\%; thus, none of these diagnostic tests showed satisfactory performance in this study [13].

In the FIGURE-OUT study, the cCTA minimal lumen diameter (MLD) was smaller than the ICA MLD (1.3 \pm 0.5 vs. $1.5 \pm 0.4 \mathrm{~mm} ; \mathrm{p}<0.001)$, and the cCTA MLA was also smaller than the IVUS MLA ( $2.2 \pm 1.2$ vs. $\left.3.2 \pm 1.2 \mathrm{~mm}^{2} ; \mathrm{p}<0.001\right)$. Compared to \%DS, MLA showed a better diagnostic performance (fig. 3). This trend was consistent regardless of lesion location, severity of stenosis, and plaque calcification. Although some studies reported that the degree of coronary artery stenosis had a tendency to be overestimated by calcified plaque, the relationships between cCTA MLD and ICA MLD as well as between cCTA MLA and IVUS MLA were not different in the setting of calcified versus noncalcified plaque in the FIGURE-OUT study. We further analyzed the correlation between cCTA \%DS and CCTA MLA in calcified versus noncalcified plaques in lesions of the FIGURE OUT study, and there was no significant difference in both lesion subsets (correlation coefficient, -0.61 in calcified lesions and -0.63 in noncalcified lesions).

The main purpose of the FIGURE-OUT study was to investigate the functional performance of the various anatomic parameters that are most commonly used in our daily practice. To adequately understand and interpret the results and clinical implications of this study, some issues need to be addressed. First, it is important to understand the limitations and pitfalls of each modality. The study results suggest that cCTA presents higher than actual lesion severity; however, this may not be true. The diameters derived from ICA and cCTA can vary because the metrics to measure the diameter of the reference segments and MLA site are different. While IVUS generally provides accurate diameter and area measurements, the IVUS catheter may not be aligned parallel to the centerline of a lumen in large and tortuous vessels. Therefore, the cross-sectional lumen area may be oblique, thereby overestimating MLA [14]. Furthermore, a recent study comparing IVUS and OCT lumen 

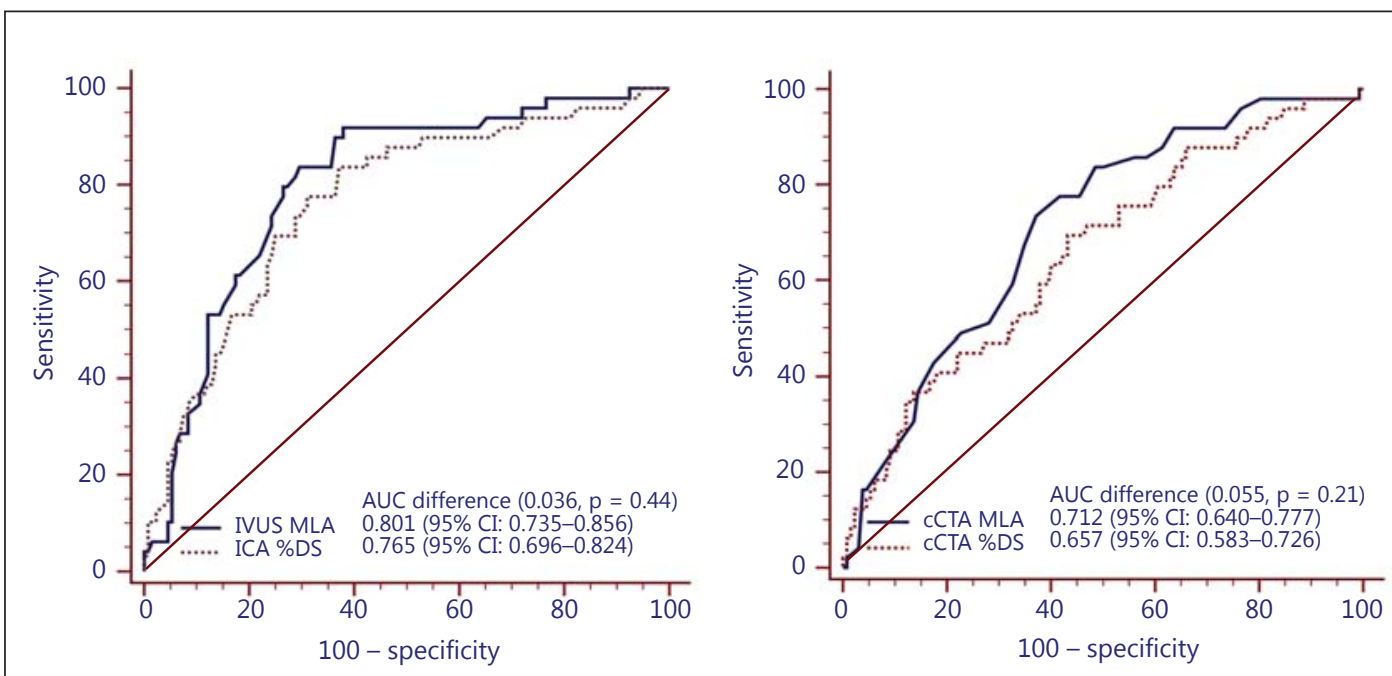

Fig. 3. Comparison of the diagnostic performance of \%DS and MLA. Comparisons between IVUS MLA versus ICA \%DS (left) and cCTA MLA versus cCTA \%DS (right) are shown. AUC = Area under the curve; CI = confidence interval.

areas showed that IVUS overestimated the true lumen area [15]. Second, the FIGURE-OUT study did not use the coregistration system for cCTA, ICA, and IVUS images. Systematic coregistration among the modalities might have decreased the difference. A dedicated registration algorithm has been developed to enable the precise matching of different imaging modalities along the longitudinal and transversal axes of coronary arteries [1618]. Finally, the recent advances in cCTA images and technology may have allowed better diagnostic performance. The study patients were enrolled between 2007 and 2011, and recently introduced CT was applied $[19,20]$. Furthermore, recently developed noninvasive FFR from CT $\left(\mathrm{FFR}_{\mathrm{CT}}\right)$, using computational fluid dynamics, consistently provides better diagnostic performance to define the functional significance of a stenosis than anatomical parameters derived from cCTA [21-23]. In the DISCOVER-FLOW study, obstructive CAD by cCTA was defined as $>50 \%$ diameter stenosis, and a hemodynamically significant lesion was defined as an FFR or $\mathrm{FFR}_{\mathrm{CT}}<0.80$ [21]. Compared to cCTA alone, $\mathrm{FFR}_{\mathrm{CT}}$ demonstrated a similar sensitivity ( 88 vs. $91 \%$ ) and negative predictive value ( 92 vs. $89 \%$ ), but there was a significantly higher specificity ( 82 vs. $40 \%$ ) and positive predictive value (74 vs. $47 \%$ ) compared to CT alone. The subsequently published DeFACTO study also demonstrated that $\mathrm{FFR}_{\mathrm{CT}}$ improved the discrimination of ischemia-causing stenoses relative to CT alone [22]. Integrated anatomical parameters such as aggregated plaque volume from cCTA may also be able to improve its diagnostic performance [24].

In conclusion, the key message from the FIGURE-OUT study is not on the matter of what is right and what is wrong. The most important lesson to be learned is that physicians should adequately understand the basic principles, limitations, and pitfalls of each modality to make an appropriate decision for the treatment of patients with CAD.

\section{Disclosure Statement}

The authors have no conflicts of interest to disclose. 
Yongzhen et al.: Noninvasive and Invasive Assessments of the Functional Significance of Intermediate Coronary Artery Stenosis: Is This a Matter of Right or Wrong?

\section{References}

1 Miller JM, Rochitte CE, Dewey M, et al: Diagnostic performance of coronary angiography by 64-row CT. N Engl J Med 2008;359:2324-2336.

-2 Min JK, Shaw LJ, Berman DS: The present state of coronary computed tomography angiography a process in evolution. J Am Coll Cardiol 2010;55:957-965.

-3 Mowatt G, Cook JA, Hillis GS, et al: 64-Slice computed tomography angiography in the diagnosis and assessment of coronary artery disease: systematic review and meta-analysis. Heart 2008;94:1386-1393.

$\checkmark 4$ Bech GJ, De Bruyne B, Pijls NH, et al: Fractional flow reserve to determine the appropriateness of angioplasty in moderate coronary stenosis: a randomized trial. Circulation 2001;103:2928-2934.

5 Pijls NH, van Schaardenburgh P, Manoharan G, et al: Percutaneous coronary intervention of functionally nonsignificant stenosis: 5-year follow-up of the DEFER Study. J Am Coll Cardiol 2007;49:2105-2111.

6 Tonino PA, De Bruyne B, Pijls NH, et al: Fractional flow reserve versus angiography for guiding percutaneous coronary intervention. N Engl J Med 2009;360:213-224.

7 De Bruyne B, Pijls NH, Kalesan B, et al: Fractional flow reserve-guided PCI versus medical therapy in stable coronary disease. N Engl J Med 2012;367:991-1001.

-8 Task Force on Myocardial Revascularization of the European Society of Cardiology (ESC) and the European Association for Cardio-Thoracic Surgery (EACTS); European Association for Percutaneous Cardiovascular Interventions (EAPCI); Kolh P, Wijns W, Danchin N, et al: Guidelines on myocardial revascularization. Eur J Cardiothorac Surg 2010;38(suppl):S1-S52.

-9 Kristensen TS, Engstrom T, Kelbaek H, von der Recke P, Nielsen MB, Kofoed KF: Correlation between coronary computed tomographic angiography and fractional flow reserve. Int J Cardiol 2010;144:200-205.

10 Papadopoulou SL, Neefjes LA, Schaap M, et al: Detection and quantification of coronary atherosclerotic plaque by 64-slice multidetector CT: a systematic head-to-head comparison with intravascular ultrasound. Atherosclerosis 2011;219:163-170.

11 Tobis J, Azarbal B, Slavin L: Assessment of intermediate severity coronary lesions in the catheterization laboratory. J Am Coll Cardiol 2007;49:839-848.

12 Christou MA, Siontis GC, Katritsis DG, Ioannidis JP: Meta-analysis of fractional flow reserve versus quantitative coronary angiography and noninvasive imaging for evaluation of myocardial ischemia. Am J Cardiol 2007;99: $450-456$.

13 Doh JH, Koo BK, Nam CW, et al: Diagnostic value of coronary CT angiography in comparison with invasive coronary angiography and intravascular ultrasound in patients with intermediate coronary artery stenosis: results from the prospective multicentre FIGURE-OUT (Functional Imaging criteria for GUiding REview of invasive coronary angiOgraphy, intravascular Ultrasound, and coronary computed Tomographic angiography) study. Eur Heart J Cardiovasc Imaging 2014;15:870-877.

14 Newby DE, Fox KA: Invasive assessment of the coronary circulation: intravascular ultrasound and Doppler. Br J Clin Pharmacol 2002;53:561-575.

15 Bezerra HG, Attizzani GF, Sirbu V, et al: Optical coherence tomography versus intravascular ultrasound to evaluate coronary artery disease and percutaneous coronary intervention. JACC Cardiovasc Interv 2013;6: 228-236.

16 Boogers MJ, Broersen A, van Velzen JE, et al: Automated quantification of coronary plaque with computed tomography: comparison with intravascular ultrasound using a dedicated registration algorithm for fusionbased quantification. Eur Heart J 2012;33:1007-1016.

17 Carlier S, Didday R, Slots T, et al: A new method for real-time co-registration of 3D coronary angiography and intravascular ultrasound or optical coherence tomography. Cardiovasc Revasc Med 2014;15:226-232.

18 Woo J, Slomka PJ, Dey D, et al: Automated multi-modality registration of 64-slice coronary CT angiography with myocardial perfusion SPECT. Proc IEEE Int Symp Biomed Imaging 2009:358-361.

19 Funabashi N, Uehara M, Takaoka H, et al: A two center 320 slice CT study for evaluating coronary arteries in subjects with chronic atrial fibrillation: a comparison of prospective and retrospective ECG-gating acquisition. Int J Cardiol 2014;177:374-379.

-20 Lee SK, Jung JI, Ko JM, Lee HG: Image quality and radiation exposure of coronary CT angiography in patients after coronary artery bypass graft surgery: influence of imaging direction with 64-slice dual-source CT. J Cardiovasc Comput Tomogr 2014;8:124-130.

21 Koo BK, Erglis A, Doh JH, et al: Diagnosis of ischemia-causing coronary stenoses by noninvasive fractional flow reserve computed from coronary computed tomographic angiograms. Results from the prospective multicenter DISCOVER-FLOW (Diagnosis of Ischemia-Causing Stenoses Obtained Via Noninvasive Fractional Flow Reserve) study. J Am Coll Cardiol 2011;58:1989-1997.

22 Min JK, Leipsic J, Pencina MJ, et al: Diagnostic accuracy of fractional flow reserve from anatomic CT angiography. JAMA 2012;308:1237-1245.

23 Norgaard BL, Leipsic J, Gaur S, et al: Diagnostic performance of noninvasive fractional flow reserve derived from coronary computed tomography angiography in suspected coronary artery disease: the NXT trial (Analysis of Coronary Blood Flow Using CT Angiography: Next Steps). J Am Coll Cardiol 2014;63:1145-1155.

-24 Nakazato R, Shalev A, Doh JH, et al: Aggregate plaque volume by coronary computed tomography angiography is superior and incremental to luminal narrowing for diagnosis of ischemic lesions of intermediate stenosis severity. J Am Coll Cardiol 2013;62:460-467. 